\section{ALTERED INTERVAL TIMING AS A NOVEL MARKER OF COGNITIVE FLUCTUATIONS IN LEWY BODY DEMENTIA}

${ }^{1}$ Elie Matar*, 'Kaylena Ehgoetz Martens, ${ }^{2} J o s e p h$ Phillips, ${ }^{1}$ Glenda Halliday, ${ }^{1}$ Simon Lewis. ${ }^{1}$ Brain and Mind Centre, University of Sydney, Camperdown, NSW, Australia; ${ }^{2}$ School of Social Sciences and Psychology, University of Western Sydney, Sydney, NSW, Australia

10.1136/jnnp-2019-anzan.1

Introduction Cognitive fluctuations are a core clinical feature of Dementia with Lewy Bodies(DLB), characterized by marked spontaneous variations in cognitive abilities and alertness. There is a paucity of objective measurements of fluctuations in the clinical setting. Altered time awareness represents a potential clinical marker of fluctuations and/or their severity. In this study we aimed to investigate qualities of interval timing in patients with DLB.

Methods 25 patients with probable DLB and 14 older controls underwent testing using a simple time perception paradigm testing probing different aspects of interval timing including time estimation(retrospective estimation of interval length), time production(prospective determination of an interval) and time pacing(explicit timing of an interval).Intervals of 10 to $90 \mathrm{~s}$ were randomized between trials.Self/carerreporting of fluctuations were measured using the clinician assessment of fluctuation(CAF) and one-day fluctuation(OFS) scales.

Results We found significant differences in interval timing between controls and DLB for time estimation and time production. Overall, DLB patients estimated less time which was significant at 90 seconds(proportion of interval $=0.92$ vs 0.69 ; $\mathrm{p}=0.03$ ). DLB produced less time(proportion of $90 \mathrm{~s}$ interval 0.58 vs $1.0 ; \mathrm{p}<0.001)$. Errors in time estimation at $90 \mathrm{sec}-$ onds correlated with fluctuation presence according to the $\mathrm{CAF}(\mathrm{r}=0.47 ; \mathrm{p}=0.009)$ whilst errors in time pacing at 90 s correlated strongest with fluctuation severity according to the OFS $(r=0.65, p<0.001)$. ROC analysis identified time production(90s) as a good test to distinguish DLB from controls (AUC $=0.8 ; 95 \%$ CI:0.75-0.98).

Conclusion We demonstrate objective evidence for altered temporal processing in DLB and suggest abnormal interval timing as a novel and clinically useful bedside marker of cognitive fluctuations.

\section{THERAPEUTIC LAG IN RELAPSING MULTIPLE SCLEROSIS}

${ }^{1,2}$ Izanne Roos*, ${ }^{3}$ Federico Frascoli, ${ }^{4,5}$ Jeannette Lechner-Scott, ${ }^{6}$ Pamela McCombe, ${ }^{7,8}$ Richard Macdonell, ${ }^{2,9,10}$ Helmut Butzkueven, ${ }^{1}$ Charles Malpas, ${ }^{1,2}$ Tomas Kalincik. ${ }^{1}$ CORe Unit, Department of Medicine, University of Melbourne, Melbourne, VIC, Australia; ${ }^{2}$ Department of Neurology, Royal Melbourne Hospital, Melbourne, VIC, Australia; ${ }^{3}$ Department of Mathematics, Swinburne University of Technology, Faculty of Science, Engineering and Technology, Melbourne, VIC, Australia; ${ }^{4}$ School of Medicine and Public Health, University of Newcastle, Newcastle, NSW, Australia; ${ }^{5}$ Department of Neurology, John Hunter Hospital, Hunter New England Health, Newcastle, NSW, Australia; ${ }^{6}$ Department of Neurology, Royal Brisbane and Women's Hospital, Brisbane, QLD, Australia; ${ }^{7}$ Department of Neurology, Austin Health, Melbourne, VIC, Australia; ${ }^{8}$ Florey Institute of Neuroscience, Melbourne, VIC, Australia; ${ }^{9}$ Department of Neuroscience, Central Clinical School, Monash University, Melbourne, VIC, Australia; ${ }^{10}$ Department of Neurology, Alfred Hospital, Melbourne, VIC, Australia

\subsection{6/jnnp-2019-anzan.2}

Introduction In multiple sclerosis (MS), treatment start or switch is prompted disease activity, often represented by relapses. Immunomodulatory therapies have potent effects on relapse rates but the time required to attain maximal effect is unclear. We aim to develop a method that allows identification of the time to full clinically manifest effect of treatment on relapses.

Methods Data from MSBase, a multinational MS registry, were used. Inclusion criteria consisted of patients with remitting relapsing MS or clinically isolated syndrome (CIS), minimum 3-year pre-treatment follow up, 1-year treatment persistence, yearly review and availability of the minimum dataset. Stratified by therapy, density curves representing relapses occurrence were created. The first local minimum of the first derivative after treatment start was identified, representing stabilisation of treatment effect. Similar method was utilised to calculate the last pre-treatment point of stabilisation. Annualised relapse rates (ARR) were compared in the pre-treatment pre stabilisation and post-treatment post stabilisation periods.

Results 4979 eligible patients with 6218 treatment epochs were identified for analysis. Time, in years, to treatment effect was shortest for interferon beta-1a sc $(0.22,0.19-0.22)$, interferon beta- $1 \mathrm{~b}(0.24,0.21-0.24)$ and fingolimod $(0.26,0.23-$ $0.26)$ and longest for dimethyl fumarate $(0.54,0.51-0.54)$ and glatiramer acetate $(0.62,0.60-0.62)$. Significant differences in pre vs post treatment ARR were present for patients on natalizumab, fingolimod and dimethyl fumarate. A sequential analysis confirmed outcome stability after approximately 1000 recorded number of events.

Conclusions We have developed a method to objectively quantify time from commencing therapy to its full effect. Time to full effect varies among therapies.

\section{SUBTHALAMIC NUCLEUS DEEP BRAIN STIMULATION EVOKED RESONANT NEURAL ACTIVITY PREDICTS CLINICAL RESPONSE TO DBS}

${ }^{1,3,2}$ San San $\mathrm{Xu}^{*}{ }^{1,2}$ Nicholas C Sinclair, ${ }^{1,4,5}$ Kristian J Bulluss, ${ }^{1,2}$ Thushara Perera, ${ }^{1,2}$ WeeLih Lee, ${ }^{1,2}$ Hugh J McDermott, $1,3,6,7$ Wesley Thevathasan. ${ }^{1}$ Bionics Institute, East Melbourne, VIC, Australia; ${ }^{2}$ Department of Medical Bionics, The University of Melbourne, Parkville, VIC, Australia; ${ }^{3}$ Department of Neurology, Austin Hospital, Heidelberg, VIC, Australia; ${ }^{4}$ Department of Neurosurgery, Austin Hospital, Heidelberg, VIC, Australia; ${ }^{5}$ Department of Neurosurgery, St Vincent's Hospital, Fitzroy, VIC, Australia; ${ }^{6}$ Department of Neurology, Royal Melbourne Hospital, Parkville, VIC, Australia; 'Department of Medicine, The University of Melbourne, Parkville, VIC, Australia

10.1136/jnnp-2019-anzan.3

Introduction DBS can improve motor deficit in Parkinson's disease (PD) patients. Existing devices have limitations due to electrode positioning errors, fallible manual programming and delivery of continuous 'open-loop' stimulation despite fluctuating patient state. This results in partial efficacy, adverse effects and increased cost. One solution is to use an electrical feedback signal or 'biomarker' recorded from DBS electrodes. The most widely studied signal has been spontaneous local field potentials (LFPs), particularly beta band (13-30 Hz) and high frequency oscillations (HFO) $(200-400 \mathrm{~Hz})$. Here, we report a novel biomarker in the form of a large amplitude, evoked potential, with a characteristic oscillatory decay, termed evoked resonant neural activity (ERNA). ${ }^{1}$

Methods LFPs and ERNA were recorded in 14 patients with PD (28 hemispheres) undergoing STN DBS surgery. The four contacts in each electrode array were ranked according to ERNA amplitude, beta power, HFO power and proximity to the anatomically ideal stimulation location. At least 3 months after surgery, motor scores (UPDRS III, reaction time) were 
evaluated off-DBS and during stimulation delivered through each electrode contact in a randomised order.

Results ERNA amplitude, beta power and contact proximity to the anatomically ideal stimulation location predicted magnitude of therapeutic response to DBS. However, after exclusion of covariance, ERNA amplitude remained the only significant predictor of DBS response.

Conclusion ERNA is a readily recordable, large amplitude signal that accurately correlates with motor response to DBS. It holds significant potential as a biomarker for guiding electrode implantation, ideal contact selection, automated parameter fitting and delivery of closed-loop DBS.

\section{REFERENCE}

1. Sinclair NC, McDermott HJ, Bulluss KJ, Fallon JB, Perera T, Xu SS, et al. Subthalamic nucleus deep brain stimulation evokes resonant neural activity. Annals of neurology 2018;83(5).

\section{VESTIBULAR EVENT MONITORING IN THE EMERGENCY DEPARTMENT}

1,2 2 Benjamin Nham*, ${ }^{1}$ Nicole Reid, ${ }^{1,2}$ Emma Argaet, ${ }^{1,2}$ Allison Young, ${ }^{3,2}{ }^{2}$ Kendall Bein,
${ }^{1,2}$ Gabor M Halmagyi, 1,2 Miriam S Welgampola. 'Institute of Clinical Neurosciences, Royal
Prince Alfred Hospital, Sydney, NSW, Australia; ${ }^{2}$ Central Clinical School, University of
Sydney, Camperdown, NSW, Australia; ${ }^{3}$ Emergency Medicine, Department of Emergency
Medicine, Royal Prince Alfred Hospital, Camperdown, NSW, Australia

10.1136/jnnp-2019-anzan.4

Introduction Acute vertigo is often accompanied by ictal-nystagmus which may assist with diagnosis. We examine the merits of a structured assessment combined with vestibular eventmonitoring in the Emergency Department (ED).

Methods We undertook a structured clinical assessment and video-nystagmography in 220 non-consecutive patients presenting to a public-hospital ED with acute vertigo, during a 10month period. The records of 115 consecutive vertiginous patients who underwent standard-assessment were compared.

Results For the structured assessment group: 54\% presented with acute vestibular syndrome (AVS), 24\% with episodic spontaneous vertigo (EVS), and 20\% with recurrent positionalvertigo (RPV).

For AVS $(n=119)$, most common diagnoses were vestibular neuritis (34\%), stroke (34\%) and vestibular migraine (13\%). Nystagmus slow-phase velocity (SPV) for VN, stroke and VM were $11 \pm 5.5 \%, 5.6 \pm 2.5 \%, 5.4 \pm 5.9 \%$; Mean ipsilesional video-head impulse gains were $0.51 \pm 0.29,0.89 \pm 0.20$ and $0.96 \pm 0.13$. For $\operatorname{EVS}(n=53)$, diagnoses included vestibular migraine (63\%), Meniere's Disease (11\%) and others (26\%). Nystagmus SPV was $5.4 \pm 3.6 \%, 7.6 \pm 6.3 \%, 4.1 \pm 1.5 \%$. In RPV $(n=43)$, common diagnoses were posterior-canal BPPV (66\%), horizontal-canal BPPV (23\%), migraine (7\%). Positional nystagmus SPV profile showed Peak SPV of $42.5 \%$, $77.6 \%$, $20.64 \%$ and Time-constants of $6.52 \mathrm{~s}, 22.51 \mathrm{~s}, 34.56 \mathrm{~s}$ for Posterior-canal BPPV, Horizontal-canal BPPV and Atypical Positional-Vertigo. A final diagnosis was reached in $96 \%$ of patients.

In the ED control group, only $77 \%$ were separated into spontaneous or positional-vertigo. A diagnosis was provided in $57 \%$ and was concordant with the history and examination in $34 \%$.

Conclusion Vestibular event-monitoring and structured clinical assessment secured a diagnosis in $96 \%$ of cases compared with 34\% for the control group, reinforcing its merit.

\section{ELECTROCLINICAL CHARACTERISTICS OF AUTOIMMUNE ENCEPHALITIS AS OUTCOME BIOMARKERS}

${ }^{1,2}$ Robb Wesselingh*, ${ }^{1,2,3}$ James Broadley, ${ }^{4,5}$ Chris Kyndt, ${ }^{4,5}$ Katherine Buzzard, 1,2Terence O'Brien, ${ }^{1,2,4}$ Mastura Monif. ${ }^{1}$ Neurosciences, Monash University, Melbourne, VIC, Australia; ${ }^{2}$ Neurosciences, Alfred Health, Melbourne, VIC, Australia; ${ }^{3}$ Neurosciences, Monash Health, Melbourne, VIC, Australia; ${ }^{4}$ Neurosciences, Melbourne Health, Melbourne, VIC, Australia; ${ }^{5}$ Neurosciences, Eastern Health, Melbourne, VIC, Australia

\subsection{6/jnnp-2019-anzan.5}

Introduction Seizures are a common characteristic of Autoimmune encephalitis (AIE). The use of the electroclinical characteristics to assist in the diagnosis of AIE has been explored $^{1}$ however use of specific electroencephalogram (EEG) changes has not been examined with regards to outcome prediction.

Methods Patients with AIE were recruited retrospectively across 4 hospitals in Victoria. Clinical Data was collected during admission and at final follow-up. EEGs of patients were reviewed using an objective proforma. Associations between EEG biomarkers and clinical outcomes were demonstrated using logistic regression modelling.

Results We recruited 88 patients with AIE and available EEGs. Presence of rhythmic delta, superimposed fast activity and an abnormal background were significantly more common in $\mathrm{N}$ methyl-D-aspartame receptor (NMDAR) antibody associated AIE patients $(\mathrm{p}<0.05)$. ICU admission was associated with rhythmic delta epileptiform activity (OR 3.25, $\mathrm{p}=0.046$ ), sharp elements in the EEG abnormality (OR 3.55, $\mathrm{p}=0.05$ ), and an abnormal background rhythm (OR 3.56, $\mathrm{p}=0.03$ ). Development of drug resistant epilepsy was associated with prolonged duration of abnormality on EEG (OR 11.99, $\mathrm{p}=0.013$ ), and sharp elements in the EEG abnormality (OR 7.29, $\mathrm{p}=0.02$ ).

Conclusion We have identified EEG biomarkers that differentiate NMDAR AIE from other subtypes, and likely represents an objective description of extreme delta brush which has previously been described in NMDAR AIE. ${ }^{2}$ We have also demonstrated biomarkers associated with important outcomes that can be used to help guide treatment and prognosis.

\section{REFERENCES}

1. Limotai C, Denlertchaikul C, Saraya AW, Jirasakuldej S. Predictive values and specificity of electroencephalographic findings in autoimmune encephalitis diagnosis. Epilepsy Behav 2018;84:29-36.

2. Veciana M, Becerra JL, Fossas P, Muriana D, Sansa G, Santamarina E, et al. EEG extreme delta brush: An ictal pattern in patients with anti-NMDA receptor encephalitis. Epilepsy Behav 2015;49:280-5.

\section{CAN SEIZURE-RELATED HEART RATE DIFFERENTIATE EPILEPTIC SEIZURES FROM PSYCHOGENIC NON- EPILEPTIC SEIZURES?}

${ }^{1}$ Hue Mun Au Yong*, ${ }^{1}$ Erica Minato, ${ }^{2}$ Eldho Paul, ${ }^{1,3}$ Udaya Seneviratne. 'Neurology Department, Monash Health, Clayton, VIC, Australia; ${ }^{2}$ Monash Centre for Health Research and Implementation, School of Public Health and Preventive Medicine, Monash University, Clayton, VIC, Australia; ${ }^{3}$ Department of Medicine, St Vincent's Hospital, University of Melbourne, Melbourne, VIC, Australia

\subsection{6/jnnp-2019-anzan.6}

Introduction This study aims to (i)evaluate the diagnostic sensitivity, specificity and predictive values of seizure-related heart rate $(\mathrm{HR})$ in differentiating epileptic seizures(ES) from 